\title{
Knowledge evaluation of preventative measures for arboviruses among pregnant women, cared for at the Santos Dumont Institute, in the Northeast of Brazil
}

\author{
Ana C. Pontes; Carolina T. R. Barreto; Carolina A. D. Santos; Larissa C. C. Rodrigues; Josélio M. G. \\ Araújo; Reginaldo A. O. Freitas Jr.
}

BACKGROUND

In 2014, the introduction of two arboviruses occurred in Brazil: Chikugunya and Zika. The primary route of transmission of these diseases is the Aedes Aegypti mosquito. However, there is increasing evidence that sexual transmission of Zika virus is possible and more common than previously predicted. Zika virus infection is a major concern among pregnant women because it is associated with fetal malformations, such as microcephaly, and Guillain-Barré syndrome.

METHODS

A questionnaire was taken by pregnant women receiving high-risk prenatal care at the Santos Dumont Institute, in the city of Macaíba, evaluating the knowledge and use of the measures recommended by the Centers for Disease Control and Prevention (CDC) for arbovirus prevention.

RESULTS

A total of 69 pregnant women were interviewed in the months of March and April 2017. The mean age was 26 years and the predominant level of education was primary education (42\%). Family income was less than four minimum wages in all interviewees. Ninety-eight percent reported knowing some prevention measures, with repellent use being the most remembered (79\%), followed by control of mosquito breeding sites $(75 \%)$.
Only $1 \%$ remembered condom use. Ninety-two percent reported using some measure during pregnancy. When asked specifically about each, $57 \%$ used repellent, $86 \%$, control of breeding grounds, 33\%, screens / mosquito nets, 34\%, long clothing and $18 \%$, condoms. Only one had exanthematic disease during pregnancy.

Percentage of patients who knew vs. used preventive measures for Zika Virus

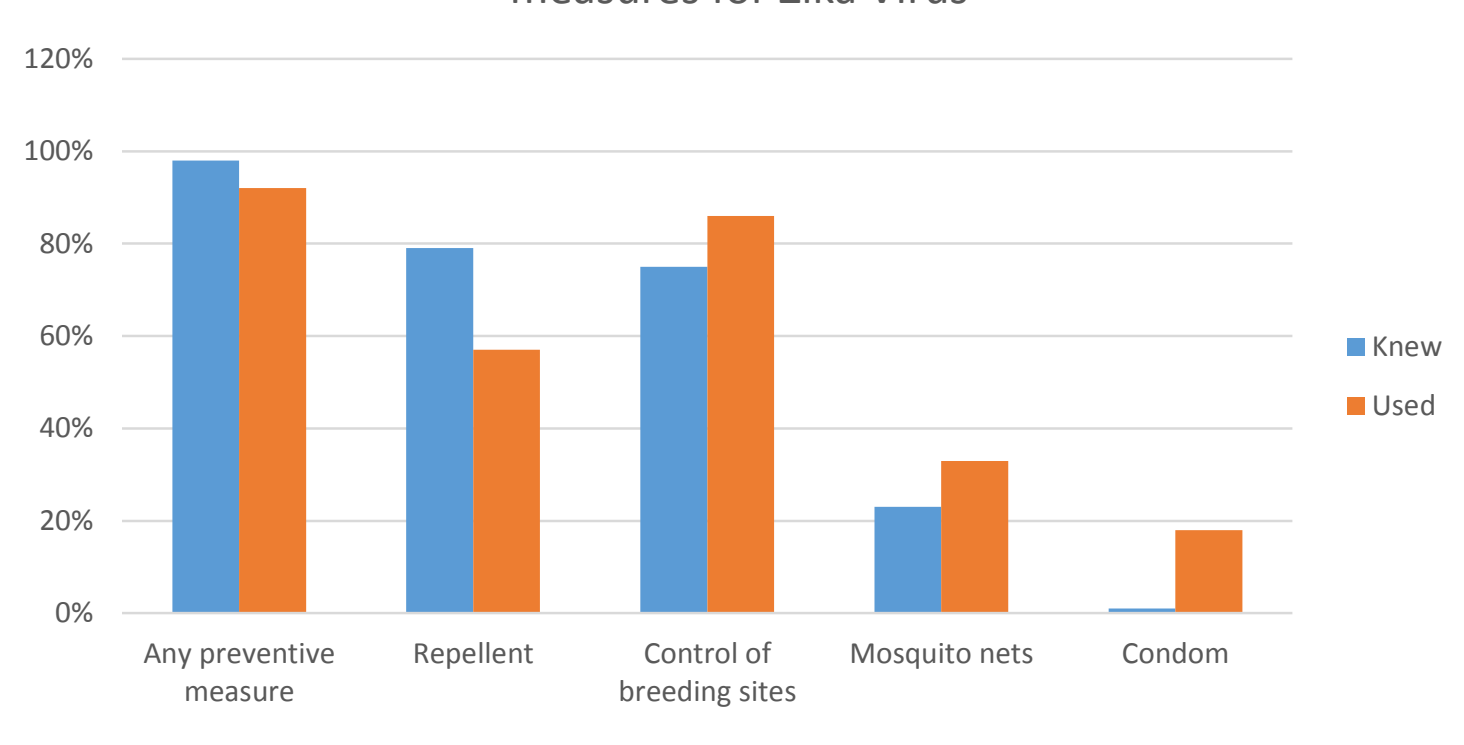

CONCLUSIONS

Most of the patients recognized the use of repellents and the control of breeding sites as a form of arbovirus prevention, although they did not adopt these measures routinely. We note that there is a lack of awareness of other less publicized means of prevention, especially the use of condoms. Thus, access to information and the empowerment of the population through continuing education are key to controlling Zika virus transmission.

Source: Gregory CJ, Oduyebo T, Brault AC, et. al. Modes of Transmission of Zika Virus. The Journal of Infectious Diseases.
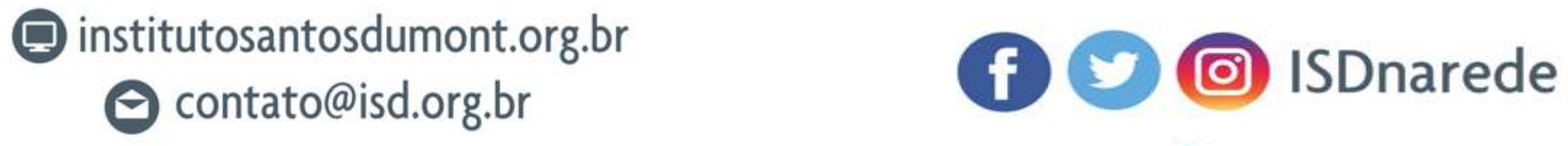